ARTICLE

https://doi.org/10.1038/s41467-021-22870-1

OPEN

\title{
Inner hair cell stereocilia are embedded in the tectorial membrane
}

\author{
Pierre Hakizimana (1) ${ }^{1 凶} \&$ Anders Fridberger (10 ${ }^{1}$
}

Mammalian hearing depends on sound-evoked displacements of the stereocilia of inner hair cells (IHCs), which cause the endogenous mechanoelectrical transducer channels to conduct inward currents of cations including $\mathrm{Ca}^{2+}$. Due to their presumed lack of contacts with the overlaying tectorial membrane (TM), the putative stimulation mechanism for these stereocilia is by means of the viscous drag of the surrounding endolymph. However, despite numerous efforts to characterize the TM by electron microscopy and other techniques, the exact IHC stereocilia-TM relationship remains elusive. Here we show that $\mathrm{Ca}^{2+}$-rich filamentous structures, that we call $\mathrm{Ca}^{2+}$ ducts, connect the TM to the IHC stereocilia to enable mechanical stimulation by the TM while also ensuring the stereocilia access to TM Ca ${ }^{2+}$. Our results call for a reassessment of the stimulation mechanism for the $\mathrm{IHC}$ stereocilia and the $\mathrm{TM}$ role in hearing.

\footnotetext{
${ }^{1}$ Department of Biomedical and Clinical Sciences (BKV), Linköping University, Linköping, Sweden. ${ }^{凶}$ email: pierre.hakizimana@liu.se
} 
S ound transduction into nerve signals happens when soundevoked vibrations of the stereocilia of the inner hair cells (IHCs) and outer hair cells (OHCs) cause an indiscriminate entry of $\mathrm{K}^{+}$and $\mathrm{Ca}^{2+}$ into the cells through open mechanoelectrical transducer (MET) channels ${ }^{1-5}$. These currents activate the OHC-based cochlear amplifier, essential for hearing faints sounds ${ }^{6,7}$ and cause the IHCs to produce electrical discharges in the auditory nerve ${ }^{8,9}$.

Patch-clamp recordings from isolated hair cells have established that extracellular $\left[\mathrm{Ca}^{2+}\right]$, which a recent discovery of high $\left[\mathrm{Ca}^{2+}\right]$ in the tectorial membrane $(\mathrm{TM})^{10}$ helped estimate at $40-150 \mu \mathrm{M}^{11}$, contributes to the regulation of hearing sensitivity.

Because the stereocilia of OHCs are attached to the $\mathrm{TM}^{12}$, their motions are proportional to the basilar membrane (BM) displacements ${ }^{13,14}$.

However, the IHC stereocilia stimulation mechanism is poorly understood.

Comparison of cochlear microphonic (CM) recordings in $\mathrm{OHC}$-less and control guinea pigs in the early 70 s suggested that the IHC stereocilia must be freestanding from the TM because their responses depended on the BM velocity ${ }^{15,16}$.

This generally agrees with the intracellular recordings from the IHCs that showed phase difference relative to CM recordings ${ }^{17}$. However, the obvious constraints imposed by the insertion of the recording electrodes ${ }^{18}$ might complicate the interpretation of these data.

While electron micrographs of the organ of Corti (OC) from the guinea pig freeze-dried samples have showed a good tissue preservation, the TM was detached from both the OHC and IHC stereocilia $^{19}$. Today, the preservation of the TM-stereocilia relationship in fixed tissues remains a challenge ${ }^{20}$.

Here we use reflected light and fluorescence confocal microscopy to investigate the TM-stereocilia relationship in the guinea pig. We show that IHC and OHC stereocilia are similarly TMembedded and that $\mathrm{Ca}^{2+}$ ducts connect the TM to the stereocilia of IHCs and OHCs.

Our results challenge the classic anatomical representation of the $\mathrm{OC}^{21}$ and show that the TM-embedment enables the stereocilia of OHCs and IHCs to directly access the TM mechanical stimulation and $\mathrm{Ca}^{2+}$.

\section{Results}

IHC stereocilia are TM-embedded. Pioneered in the $80 \mathrm{~s}^{22}$, the temporal bone preparation from the guinea pig makes it possible to investigate sound transduction in functional and largely intact $\mathrm{OC}^{10,23-26}$. Its $\mathrm{CM}$ recordings exhibit amplitudes and nonlinearity (Fig. 1a, b, c) generally consistent with those measured in living animals ${ }^{27}$. In addition, its cochlear mechanical responses are consistent with those obtained in vivo in intact cochlea ${ }^{28}$ and in vivo OC confocal imaging ${ }^{29}$ has confirmed that the hair cell morphology is well preserved in our preparation. This suggests that the native morphological organization and physiology of the OC and the TM, are most likely preserved in this preparation, making it suitable for investigating TM-IHC stereocilia relationships.

Although the TM appears nearly completely transparent to white light ${ }^{30}$, we realized recently that it reflects the green laser light, which we used to acquire confocal images of the TM and $\mathrm{OC}$ in situ. Staining with fluorescence dyes is not necessary and therefore image acquisition can be achieved through an intact Reissner's membrane.

In the reflected light images, the reticular lamina $(\mathrm{RL})$ was brighter than the TM, making it easier to distinguish an interface line between the two structures, with no obvious gap between them (Fig. 1d). This is consistent with the fact that the stereocilia in the $\mathrm{OHC}$ region are attached to the $\mathrm{TM}^{12,31}$. However, the
TM-RL attachment, which contrasts with the traditional schematic representation of the $\mathrm{OC}^{21}$, indicates in addition that the OHC stereocilia are TM-embedded. Furthermore, the TM-RL attachment in the IHC region was surprising and suggests that the IHC stereocilia, too, are TM-embedded.

To test the TM-RL attachment strength, we stimulated the OC with a sound intensity of $103 \mathrm{~dB}$ sound pressure level (SPL), which causes the RL to move towards the $\mathrm{BM}^{32}$ (see Supplementary Movie 1). Surprisingly, the TM was pulled along while its shared interface with the RL, including in the IHC region, remained tight, causing the TM to drastically compress as it adjusted to the deformation of the OC (Supplementary Movie 1). This behavior was seen in all 37 preparations tested and indicated that the TM and RL were firmly attached in the IHC and OHC regions alike.

Immediately after the acoustic overstimulation stopped, the TM relaxed faster than the RL, causing a TM-RL gap to briefly appear (Supplementary Movie 1 and Fig. 1e), suggesting that the TM-RL attachments were overstretched by the OC contraction. However, this gap recovered (Supplementary Movie 1, compare Fig. $1 \mathrm{~d}-\mathrm{f}$ and Fig. $1 \mathrm{~g}$ vs. h), indicating that it represented rather a pathological state.

$\mathrm{Ca}^{2+}$ is required for the integrity of various sensory structures of the $\mathrm{OC}^{33,34}$ but its effect on the TM integrity is poorly documented. Swapping endolymph with perilymph affected TM thickness but the results largely varied from one study to another ${ }^{35,36}$. Millimolar concentrations of $\mathrm{Ca}^{2+}$-chelating agents are routinely used to remove the TM from the $\mathrm{RL}^{37}$, but micromolar concentrations deplete $\mathrm{TM} \mathrm{Ca}{ }^{2+}$ without detaching the TM and had a reversible effect on mechanotransduction ${ }^{10}$. This suggests that low levels of $\mathrm{Ca}^{2+}$-chelating agents could be used to investigate $\mathrm{Ca}^{2+}$ depletion effect on the TM relationships with the RL and stereocilia in functional OC.

Figure 1i and Supplementary Movie 2 show that before ethylene glycol-bis(2-aminoethylether)- $N, N, N^{\prime}, N^{\prime}$-tetraacetic acid (EGTA) application, the TM rested tightly on the RL. However, upon $100 \mu \mathrm{M}$ EGTA application through a thin glass electrode, the TM swelled rapidly, causing its normally "biconvex" shape to look more circular (Fig. 1j, Supplementary Movie 2); a behavior consistent in six preparations tested. These shape changes also caused a small TM-RL gap to appear in the OHC region (Fig. 1j, Fig. 1k vs. 1l, and Supplementary Movie 2). Although these changes were largely reversible (Supplementary Movie 2), they also suggested that $\mathrm{Ca}^{2+}$ depletion caused the TM interactions with the RL and stereocilia to weaken.

To investigate this possibility, we subjected the TM-RL junction to acoustic overstimulation in five of the preparations above immediately after EGTA application.

As shown by Supplementary Movie 2, after a brief acoustic overstimulation, the TM detached from the $\mathrm{RL}$ in all five preparations tested. Typically, when overstimulation stopped, the TM-RL attachments tended to recover (Supplementary Movie 2) but repeated EGTA injections combined with overstimulation eventually caused the TM to permanently detach from the RL, leaving the OC TM-less (Fig. $1 \mathrm{~m}$ and Supplementary Movie 2). This indicates that the TM-RL attachments were robust but also $\mathrm{Ca}^{2+}$-dependent.

To gain a deeper insight into the TM-stereocilia relationship, we combined reflected light and fluorescence confocal imaging after OC staining with the membrane dye di-3-aneppdhq ${ }^{10}$.

High-resolution reflected light confocal images showed that the TM rested on the OC, where it intimately interacted with the RL, seen as a bright structure that spans from the inner phalangeal cells to the Hensen's cells (Fig. 2a). A close inspection of the RL revealed weaker reflectivity zones in the $\mathrm{OHC}$ regions that delimited adjacent cuticular plates of the three OHC rows (Fig. 2a), in direct contact with the TM. 
CM @74dB SPL

a

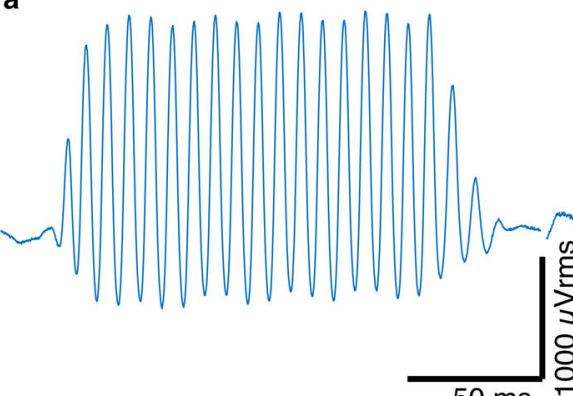

$50 \mathrm{~ms}$

b

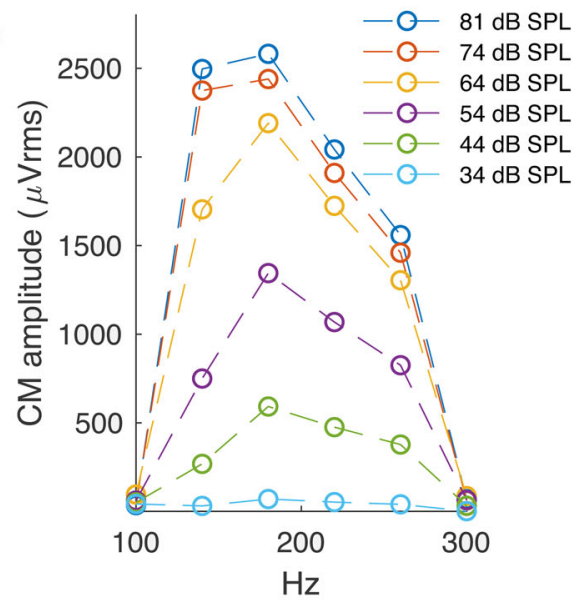

CM @54dB SPL

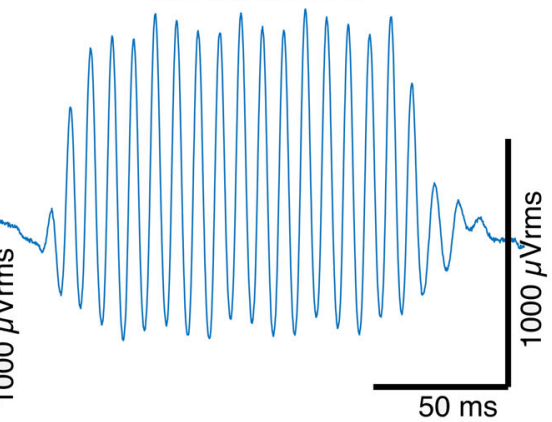

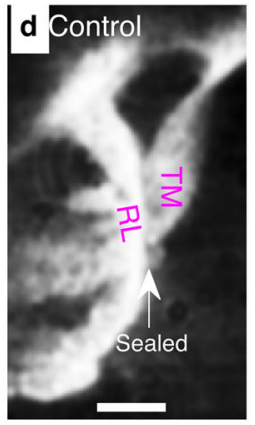
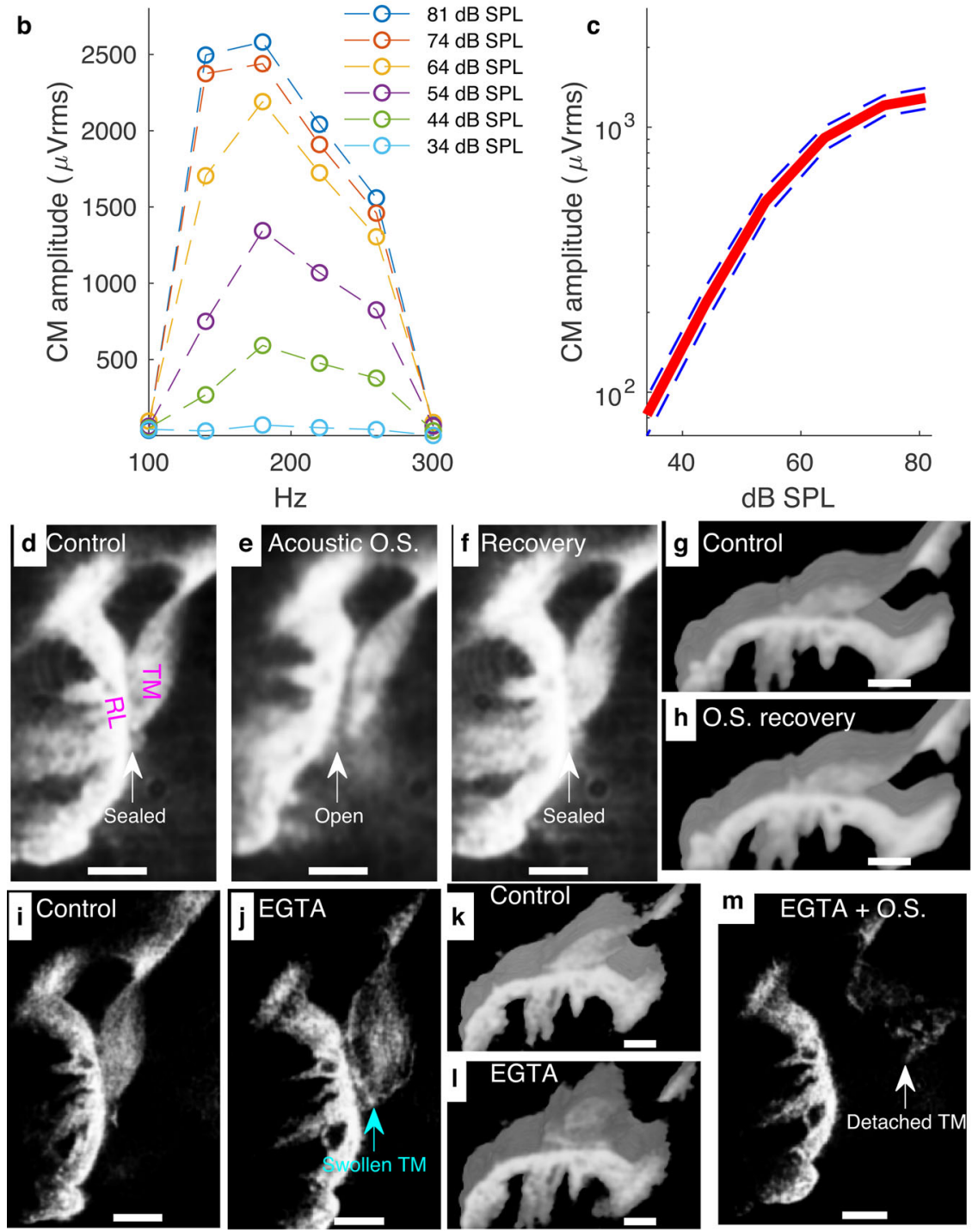

Surprisingly, the head region of the pillar cells was poorly reflective (Fig. 2a). A survey of the literature showed that this region is highly enriched in actin filaments ${ }^{38}$, a characteristic shared with the stereocilia ${ }^{38}$. This similarity could explain why both structure types had a poor reflectivity (Fig. 2a).

In the fluorescence channel, di-3-aneppdhq-stained the hair cells and their stereocilia ${ }^{10}$ (Fig. 2b).

Overlay of the reflected light and fluorescence channels showed that the OHC somatic and cuticular plate signals from both channels matched (Fig. 2c, d). In addition, the overlay demonstrated that the IHC stereocilia were TM-embedded (Fig. 2d). The image also shows that the $\mathrm{OHC}$ stereocilia were not merely attached to the TM but were rather TM-embedded (Fig. 2c).

$\mathrm{Ca}^{2+}$ ducts ensure stereocilia access to $\mathbf{T M} \mathbf{C a}^{2+}$. To further investigate the TM-stereocilia relationships, we used a $\mathrm{Ca}^{2+}$ ratiometric indicator where Cal-520L, a bright and sensitive low affinity $\mathrm{Ca}^{2+}$ dye, and $\mathrm{Cy} 5$, a bright $\mathrm{Ca}^{2+}$-insensitive fluorophore, were conjugated to a $10 \mathrm{kDa}$-dextran. Because Cal-520L/Cy5 were spectrally independent, their presence made the conjugate a reliable ratiometric $\mathrm{Ca}^{2+}$ indicator. Typically, 2D OC confocal 
Fig. 1 The TM interacts tightly with the RL including in the IHC region. a-c Effect of sound pressure level (SPL) on CM amplitude in the cochlear apical region in the temporal bone preparation from the guinea pig. When the eardrum is acoustically stimulated by a speaker inserted in the ear canal, the hair cells produce the CM (the individual examples shown in a were recorded in response to a $180-\mathrm{Hz}$ tone burst at 74 and $54 \mathrm{~dB}$ SPL respectively). The peakto-peak amplitudes from the raw CM waveforms were plotted against frequency and for several dB SPL values to generate the frequency-tuning curves shown in $\mathbf{b}$ (the individual curves shown in $\mathbf{b}$ are from the same preparation as $\mathbf{a}$ ). The CM peak-to-peak amplitudes associated with the frequency-tuning curves were then averaged for 26 preparations and plotted against dB SPL to produce the plot shown in c (solid line, mean; error bar, s.e.m). Source data are provided as a Source data file. $\mathbf{d}$ Reflected light confocal image of the OC. Note the sealed TM-RL interface including within the IHC region. e Acoustic overstimulation (OS)-induced contraction of the OC exposed a TM-RL gap. $\mathbf{f}$ When the OS stopped, the TM-RL gap resealed. $\mathbf{g} 3 \mathrm{D}$ reconstruction (29 $\mathrm{z}$ stacks with a spacing of $1 \mu \mathrm{m}$ ) of the OC before acoustic overstimulation. The image was rotated to show the opposite side of the image shown in $\mathbf{a}$. $\mathbf{h}$ 3D reconstruction ( $26 z$-stacks with a spacing of $1 \mu \mathrm{m}$ ) of the OC after the preparation was allowed to recover from OS. $\mathbf{i}$ Image of the OC showing a tight TMRL interface before EGTA treatment. $\mathbf{j}$ Upon EGTA injection, the TM swelled markedly, which exposed a TM-RL gap. $\mathbf{k}$, I 3D reconstructions of the OC before (k) and after EGTA injection (I) show that the TM swelling associated with $\mathrm{Ca}^{2+}$ removal by EGTA caused the TM to loosen from the RL (both reconstructions were obtained from 41 z-stacks with a spacing of $1 \mu \mathrm{m}$ ). $\mathbf{m}$ When the EGTA injection was followed by OS, the TM detached from the RL. All scalebars $50 \mu \mathrm{m}$. Data in $\mathbf{d}-\mathbf{h}$ were representative of 37 different preparations. Data in $\mathbf{i}-\mathbf{I}$ were representative of six preparations; Data in $\mathbf{m}$ are representative of five preparations.
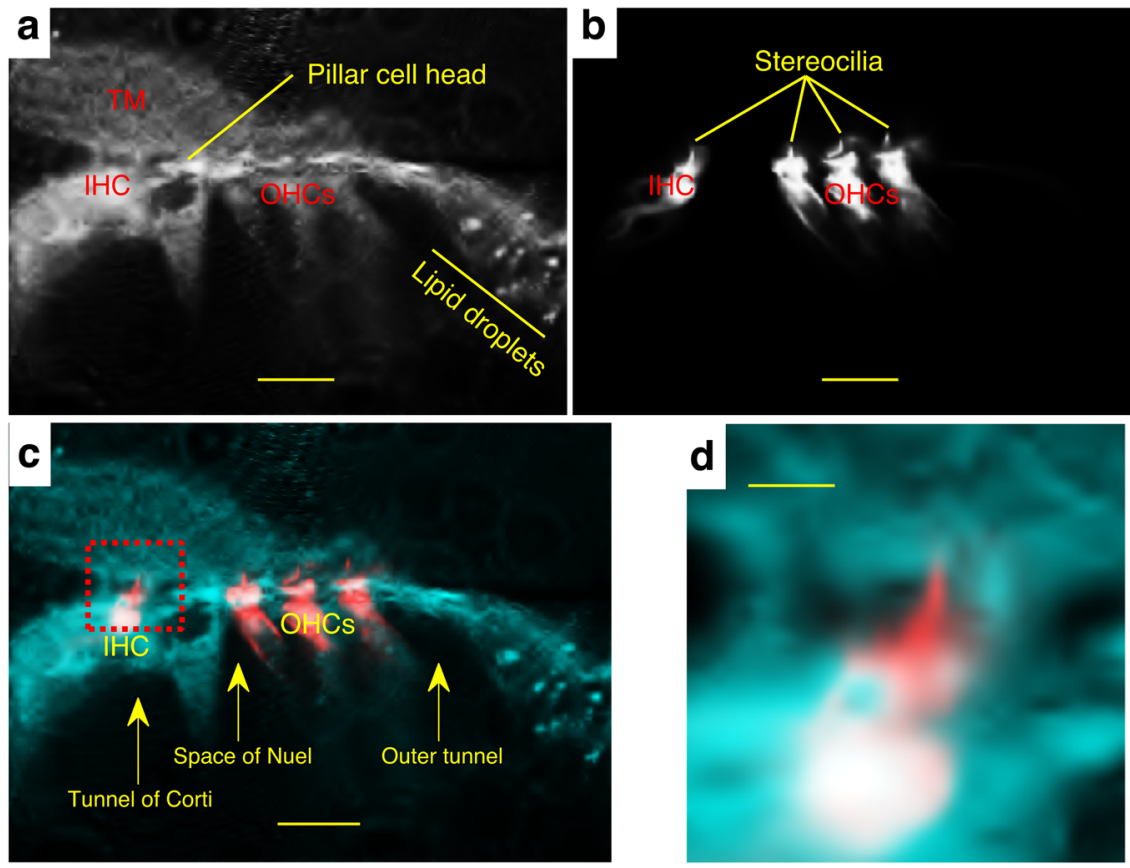

Fig. 2 IHC and OHC stereocilia bundles alike are TM-embedded. a Reflected light confocal image of the OC showing tight interaction between the TM and RL. b Fluorescence confocal image of the same focal plane as in a after staining the OC with the membrane dye di-3-aneppdhq. c Overlay of images from $\mathbf{a}$ and $\mathbf{b}$ revealed that stereocilia of $\mathrm{IHC}$ are TM-embedded in a similar fashion as the OHC stereocilia. $\mathbf{d}$ A closeup view on the IHC region (see red square in c) highlights the IHC stereocilia embedment within the TM. Scalebars: $25 \mu \mathrm{m}$ in $\mathbf{a}, \mathbf{b}$, and $\mathbf{c} ; 5 \mu \mathrm{m}$ in $\mathbf{d}$. Data in $\mathbf{a}-\mathbf{d}$ were representative of ten different preparations.

images associated with the two fluorophores were acquired simultaneously within minutes of the indicator injection.

To visualize purely $\mathrm{Ca}^{2+}$-dependent signals, the Cal-520L and Cy5 2D confocal images were divided on a pixel-by-pixel basis, which resulted in ratiometric images of the OC, where the ratio in each pixel depends on $\mathrm{Ca}^{2+}$ (Fig. 3a, b).

As shown by Fig. $3 \mathrm{a}-\mathrm{d}, \mathrm{Ca}^{2+}$ ratiometric imaging produced $\mathrm{Ca}^{2+}$ signals within the stereocilia while also revealing high $\mathrm{Ca}^{2+}$ filamentous structures, or $\mathrm{Ca}^{2+}$ ducts, that radially spanned the TM to attach to the IHC and OHC stereocilia.

Although most of these $\mathrm{Ca}^{2+}$ ducts ran parallel to each other, they also branched occasionally, especially closer to the stereocilia attachment points, where they contacted stereocilia bundles in IHCs, row 1 OHCs, row 2 OHCs, and row 3 OHCs (Fig. 3a-d) and these findings were consistent in several locations of the cochlear apical region imaged in 12 preparations.

Since the $\mathrm{Ca}^{2+}$ concentration in the stereocilia has remained elusive despite its critical importance for hearing ${ }^{11}$, we used the ratiometric images to quantify the stereocilia bundle $\mathrm{Ca}^{2+}$ ratios (Fig. 3e). In IHCs, the $\mathrm{Ca}^{2+}$ ratio was $0.94 \pm 0.02(n=19 \mathrm{IHCs}$ from 11 preparations, mean \pm s.e.m.). In row $1 \mathrm{OHCs}$, the $\mathrm{Ca}^{2+}$ ratio was $0.91 \pm 0.03$ (mean \pm s.e.m.; $n=22$ row 1 OHCs from ten preparations). In row $2 \mathrm{OHCs}$, the $\mathrm{Ca}^{2+}$ ratio was $0.88 \pm 0.03$ (mean \pm s.e.m.; $n=34$ row 2 OHCs from eight preparations). In row 3 OHCs, the $\mathrm{Ca}^{2+}$ ratio was $0.94 \pm 0.01$ (mean \pm s.e.m.; $n=$ 33 row 3 OHCs from eight preparations). The $\mathrm{Ca}^{2+}$ ratio differences between the stereocilia types were not statistically significant $(p=0.1$, Kruskal-Wallis test).

Interestingly, the $\mathrm{Ca}^{2+}$ indicator produced a signal in the cell bodies (Fig. 3a-d), possibly due to the accumulation of the indicator from the injection site (Fig. 3f) via endocytosis typical to dextrans ${ }^{39}$.

To determine whether $\mathrm{Ca}^{2+}$ from the ducts is transported to the stereocilia, we assessed $\mathrm{Ca}^{2+}$ mobility in the ducts relative to the stereocilia. Specifically, we monitored the fluorescence recovery of the dextran-conjugated $\mathrm{Ca}^{2+}$ dye Cal-520L after photobleaching (FRAP, reviewed in $^{40}$ ) a 1 micron-wide spot 
a

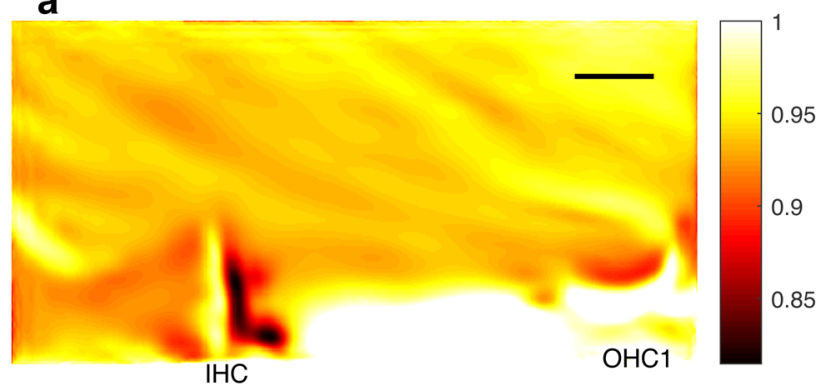

c

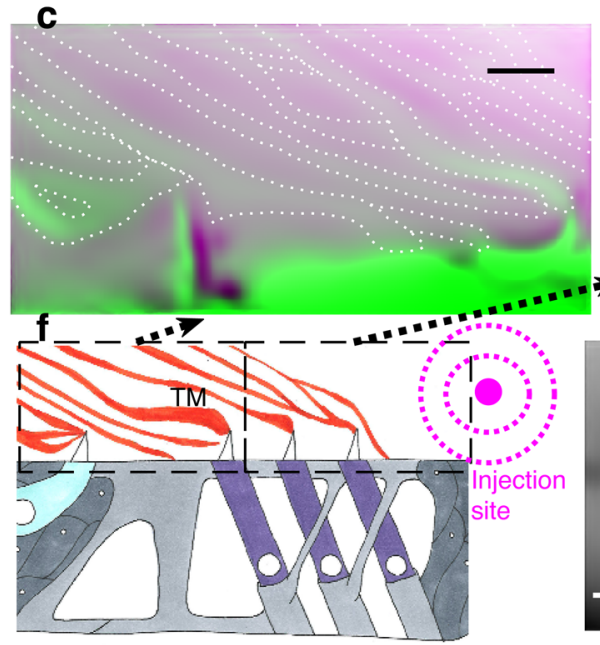

d b
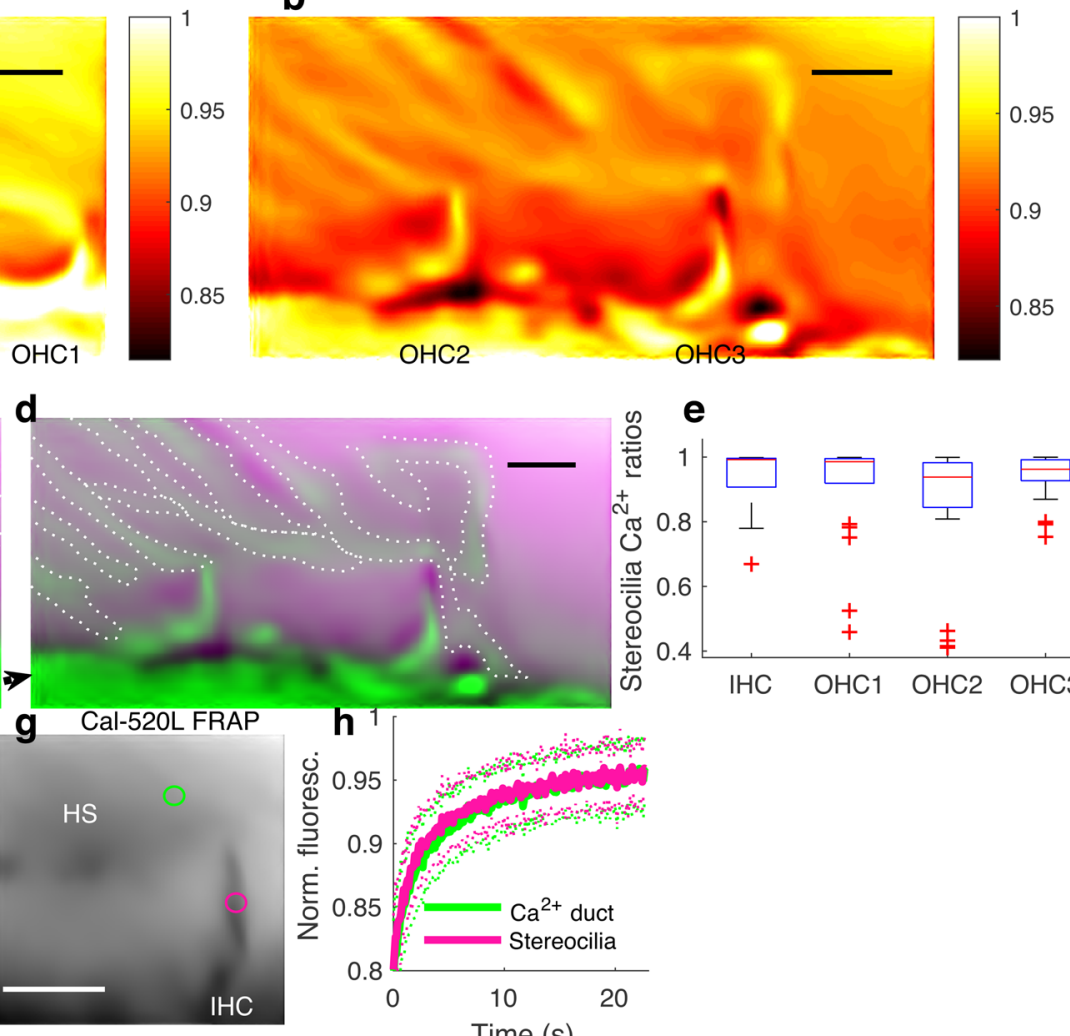

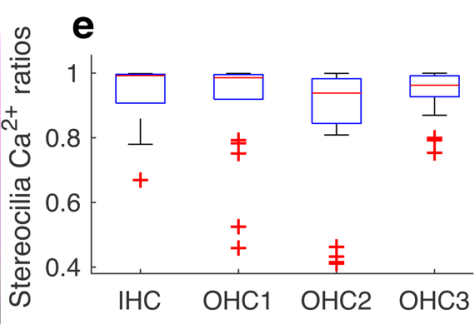

Fig. $3 \mathbf{C a}^{2+}$ ducts connect the TM to the IHC and OHC stereocilia. $\mathbf{a}, \mathbf{b} \mathrm{Ca}^{2+}$ ratios, revealed Ca ${ }^{2+}$-rich ducts that connect the $\mathrm{TM}$ to the stereocilia of IHCs

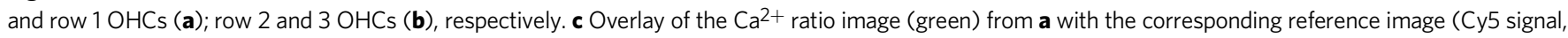
violet) to highlight the contacts between the stereocilia of the IHCs and row $1 \mathrm{OHCs}$ with the Ca ${ }^{2+}$ ducts. Dotted white lines were manually drawn to depict the boundaries of the $\mathrm{Ca}^{2+}$ ducts. $\mathbf{d}$ Same as in c, for row 2 and row $3 \mathrm{OHC}$ regions. e $\mathrm{Ca}^{2+}$ ratios in the stereocilia bundles of different hair cell types. OHC1, row 1 $\mathrm{OHCs}$; OHC2, row $2 \mathrm{OHCs}$; OHC3, row $3 \mathrm{OHCs}$. For IHCs, $n=19 \mathrm{lHCs}$ from 11 preparations; for $\mathrm{OHC1}, n=22$ row $1 \mathrm{OHCs}$ from ten preparations; for OHC2, $n$ $=34$ row $2 \mathrm{OHCs}$ from eight preparations; for $\mathrm{OHC} 3, n=33$ row $3 \mathrm{OHCs}$ from eight preparations. Box-and-whiskers plot represents the maxima, 75 th percentile, median, 25th percentile, and minima. Source data are provided as a Source data file. $\mathbf{f} \mathrm{OC}$ schematic highlighting the regions confocally imaged to obtain images shown in $\mathbf{a}-\mathbf{d}$ and injection site. $\mathbf{g}$, $\mathbf{h}$ Single channel Cal-52OL confocal image of the IHC stereocilia and the associated Ca2+ duct-rich TM region. The green and magenta ROls (g) were used to investigate $\mathrm{Ca}^{2+}$ mobility in the $\mathrm{Ca}^{2+}$ duct-rich region and the IHC stereocilia, respectively by fluorescence recovery after photobleaching (FRAP). Averaged FRAP data associated with the ROls shown in $\mathbf{h}$ (solid lines, means; error bars, s.d.). Source data are provided as a Source data file. For data in $\mathbf{g}, \mathbf{h}, n=41$ same-location TM-IHC stereocilia from five different preparations. Scalebars $5 \mu$ m.

within a $\mathrm{Ca}^{2+}$ duct-rich region and in same-location IHC stereocilia near the center of the bundle (Fig. $3 \mathrm{~g}$ ).

After photobleaching the $\mathrm{Ca}^{2+}$ signal (i.e., Cal-520L fluorescence), it recovered rapidly in both regions of interest (Fig. 3h). On average, the mobile fractions were $0.75 \pm 0.01$ and $0.76 \pm 0.02$ in the stereocilia and same-location $\mathrm{Ca}^{2+}$ duct region, respectively (mean \pm s.e.m, $n=41$ same-location TM-IHC stereocilia from five preparations). The difference was not statistically significant ( $p=0.7$, Wilcoxon signed-rank test). The recovery halftime in $\mathrm{Ca}^{2+}$ duct region was $2.89 \pm 0.11$ s (mean \pm s.e.m, $n=$ 41 same-location TM-IHC stereocilia from five preparations). For the stereocilia bundles, the recovery halftime was significantly shorter, i.e., $2.58 \pm 0.10 \mathrm{~s}$ ( $p=0.002$, Wilcoxon signed-rank test).

The high mobile fraction of as much as $75-76 \%$ suggests that much of the $\mathrm{Ca}^{2+}$ in the stereocilia and $\mathrm{Ca}^{2+}$ ducts was freely mobile while the reminder could have a role in maintaining the integrity of the TM and sensory structures as discussed above.

Assuming that some form of $\mathrm{Ca}^{2+}$ equilibrium exists between the $\mathrm{Ca}^{2+}$ ducts and the stereocilia thanks to their attachments, the faster flow of stereocilia $\mathrm{Ca}^{2+}$ into the cell would drive the ducts' $\mathrm{Ca}^{2+}$ into the stereocilia due to the Le Chatelier principle.

Together, the data described above indicate that the $\mathrm{Ca}^{2+}$ ducts attach the TM to the stereocilia of IHCs and OHCs and support that the $\mathrm{Ca}^{2+}$ ducts ensure these stereocilia access to $\mathrm{TM} \mathrm{Ca}{ }^{2+}$.

Sound-evoked mechanical responses of the TM and stereocilia. The TM-embedment of the IHC stereocilia raises the question of how these stereocilia are stimulated.

To address this issue, we stained the TM (known to have a high negative charge-density ${ }^{41}$ ), with the cationic dye di-3-aneppdhq ${ }^{10}$ and then investigated the sound-evoked motions of same-location TM and stereocilia by high-speed confocal imaging ${ }^{42}$.

Figure $4 \mathrm{a}$ shows the fluorescence pattern across the di-3aneppdhq-stained OC.

Di-3-aneppdhq-stained the hair cells and their stereocilia (Fig. 4a) whose morphology was similar to what is seen in vivo ${ }^{29}$.

Surprisingly, di-3-aneppdhq-stained also the TM (Fig. 4a), indicating that adjusting the microscope sensitivity can reveal more details in the TM (compare Figs. $4 \mathrm{a}$ and 2b). Although the TM staining was relatively weak, it was enough to see that the IHC and OHC stereocilia bundles were TM-embedded (Fig. 4a).

A close examination of the TM revealed $1 \mu \mathrm{m}$-wide granular structures (GSs) randomly distributed across the TM (Fig. 4a). Remarkably, one GS can be seen near the RL, supporting the TMRL attachment (Fig. 4a). 


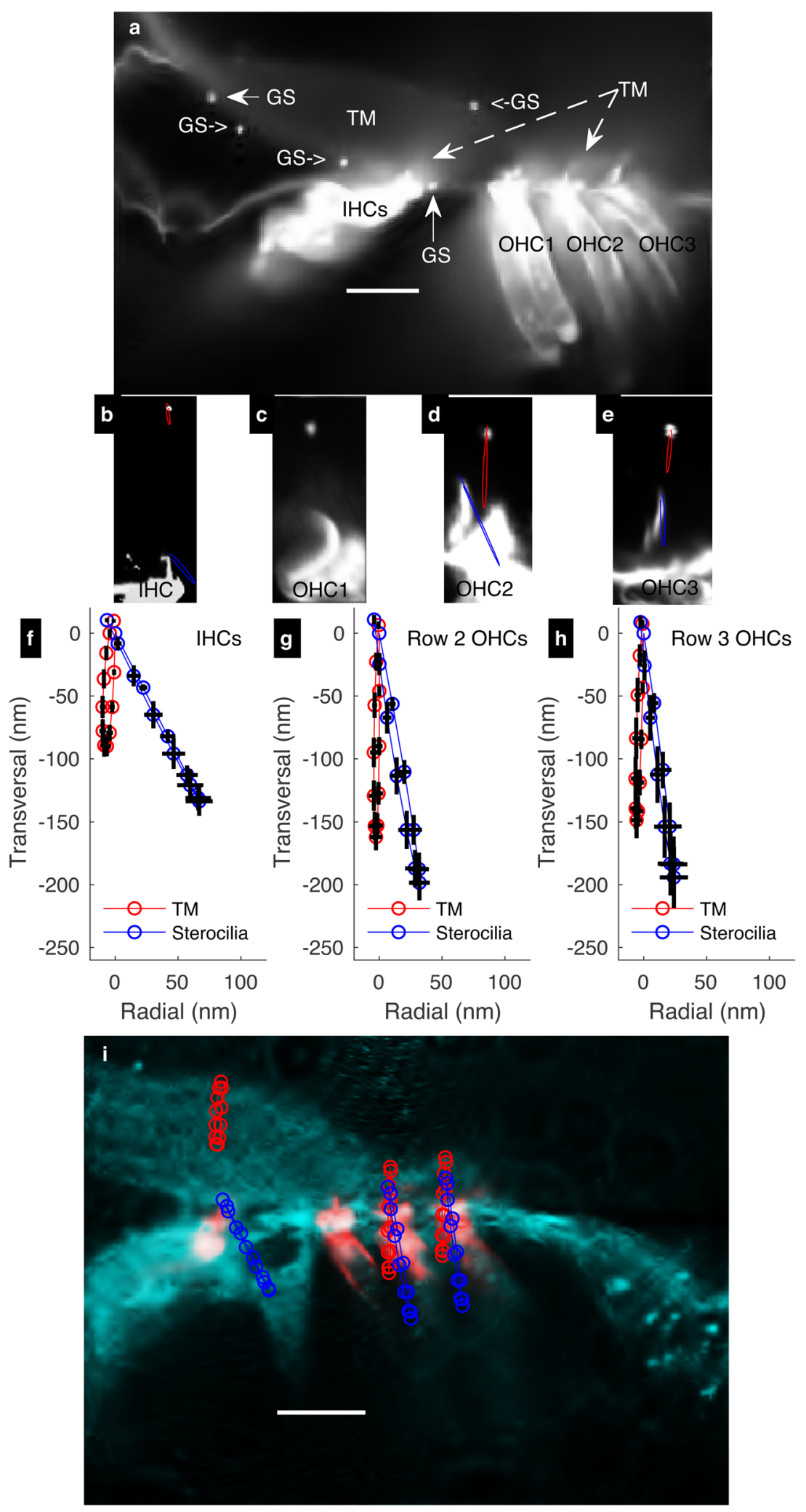

We then used GS-stereocilia bundle pairs (Fig. 4b-e) to investigate the sound-evoked mechanical relationship between the TM and stereocilia in IHC and OHC regions by high-speed confocal imaging ${ }^{42}$, which allows for acquisition of 12 images at 12 equidistant phases of the acoustic stimulus. Row 1 OHC stereocilia bundles (Fig. 4c) were not included because their stereocilia bundles do not often appear perfectly straight when viewed in the cross-section orientation.
To quantify the sound-evoked motion of the TM and stereocilia, the image series above were analysed by optical flow algorithms in MATLAB ${ }^{42}$, which tracks and stores the coordinates of these structures across the acoustic phases.

Plotting these phase-dependent positions in a twodimensional-space (transversal vs. radial directions) yield elliptical trajectories, for the TM (red trajectories, Fig. $4 \mathrm{~b}, \mathrm{~d}, \mathrm{e}$ ) and stereocilia (blue trajectories, Fig. $4 \mathrm{~b}, \mathrm{~d}, \mathrm{e}$ ) in the IHC and $\mathrm{OHC}$ 
Fig. 4 Sound-evoked movements of same-location TM and stereocilia bundles from IHCS and OHCS. a A confocal image of di-3-aneppdhq-stained OC revealed bright granular structures (GSs) scattered around the TM. These structures were used to track the sound-evoked movements of the TM. b-e. Confocal images of same-location TM and stereocilia bundles in IHCs, row $1 \mathrm{OHCs}$, row $2 \mathrm{OHCs}$, and row $3 \mathrm{OHCs}$, respectively. Example of individual sound-evoked motion trajectories associated with these structures at the best frequency (b, $\mathbf{d}$, and $\mathbf{e})$ are shown in red for the TM and blue for the stereocilia. $\mathbf{f - h}$ Sound-evoked motion trajectories at the best frequency for the TM (red) and same-location stereocilia bundle tip (bleu) in IHCs, row 2 OHCs, and row 3 OHCs, respectively as averaged in several preparations (solid line, mean; error bars, s.e.m.). For IHCs, $n=21$ same-location TM and IHCs from nine preparations; for row 2 OHCs, $n=$ 23 same-location TM and row $2 \mathrm{OHCs}$ from 12 preparations; for row $3 \mathrm{OHCs} n=17$ same-location TM and row 3 OHCs from 13 preparations. i The mean values for trajectory data from $\mathbf{f}-\mathbf{h}$ (for $n$ values, see the previous sentence) were replotted on the composite image from Fig. $2 c$ (representative of ten different preparations) to highlight the $\mathrm{OC}$ regions where the motion measurements were performed. All scalebars $25 \mu \mathrm{m}$.

regions. Trajectories from different preparations were then fitted with ellipse equations (see Methods) for statistical analysis of the associated parameters.

Figure $4 \mathrm{f}-\mathrm{h}$, show the averaged sound-evoked motion trajectories of the TM and stereocilia from the IHCs, row 2 $\mathrm{OHCs}$, and row $3 \mathrm{OHCs}$, respectively when the intensity and the frequency of the sound stimulus were respectively $\approx 80 \mathrm{~dB}$ SPL and $\approx 200 \mathrm{~Hz}$ (the best frequency, BF). It was evident that the motion amplitude of the TM was smaller relative to samelocation stereocilia. However, this behavior was a common feature in both the IHCs and OHCs as detailed below.

In the IHC region, the TM moved nearly transversally towards the BM, with a major peak-to-peak amplitude of $116 \pm$ $7 \mathrm{~nm}$ (mean \pm s.e.m., $n=21$ same-location TM and IHCs from nine preparations, red plots, Fig. $4 \mathrm{f}$ ). In the row $2 \mathrm{OHC}$ region (Fig. 4g), the TM moved with an amplitude of $178 \pm 11 \mathrm{~nm}$ (mean \pm s.e.m.; $n=23$ same-location TM and row 2 OHCs from 12 preparations) while in row $3 \mathrm{OHC}$ region (Fig. $4 \mathrm{~h}$ ) the TM motion had a peak-to-peak amplitude of $183 \pm 13 \mathrm{~nm}$ (mean \pm s.e.m.; $n=17$ same-location TM and row 3 OHCs from 13 preparations).

In agreement with past studies $24,25,43$, the motion trajectory at the stereocilia bundle tip was radially inclined and the major axis peakto-peak amplitude was larger relative to the TM across the OC (Fig. $4 \mathrm{f}-\mathrm{h}$ ). In the IHC region (Fig. 4f, blue trajectory), the peak-topeak motion amplitude of the stereocilia bundle tip was $180 \pm 13 \mathrm{~nm}$ (mean \pm s.e.m.; $n=21$ same-location TM and IHCs from nine preparations) and the difference relative to the same-location TM counterpart was significant $(p=0.00006$, Wilcoxon signed-rank test). In row 2 OHCs (Fig. $4 \mathrm{~g}$, blue trajectory), the stereocilia tip motion amplitude was $224 \pm 14 \mathrm{~nm}$ (mean \pm s.e.m.; $n=23$, samelocation TM and row 2 OHCs from 12 preparations), which was significantly larger relative to same-location TM counterpart ( $p=$ 0.00003 , Wilcoxon signed-rank test). In row $3 \mathrm{OHC}$ stereocilia (Fig. 4h, blue trajectory), the peak-to-peak motion amplitude of the stereocilia tip was $251 \pm 22 \mathrm{~nm}$ (mean \pm s.e.m.; $n=17$ same-location $\mathrm{TM}$ and row 3 OHCs from 13 preparations), which in this case was also significantly larger compared to same-location TM motion amplitude ( $p=0.0003$, Wilcoxon signed-rank test).

Plotting the average trajectories on their approximative location on the composite image of the OC from Fig. $2 c$, confirms that the peak-to-peak motion amplitude for the TM was smaller relative to same-location bundle tip, irrespective of the hair cell type (Fig. 4i). Since our data above show that the TM and stereocilia are attached, the difference in their motion amplitudes indicates that the TM-stereocilia links stretched during sound stimulation.

As shown by Fig. 5a-c, the stereocilia and TM responded to frequencies between 150 and $500 \mathrm{~Hz}$ and the motions were tuned. In addition, same-location TM and stereocilia had a similar BF, irrespective of the hair cell type. Although the BF exhibited small variations with values of $230-240 \mathrm{~Hz}$ for the IHC and row $3 \mathrm{OHC}$ regions, and $200 \mathrm{~Hz}$ for the row $2 \mathrm{OHC}$ region, this was clearly not a parameter that distinguished IHC from OHC regions (Fig. 5a-c). The differences between the TM and stereocilia motion amplitudes seen across frequency indicate that the TMstereocilia links stretched in a frequency-dependent fashion both in the IHC and $\mathrm{OHC}$ regions.

Radially, the stereocilia motion amplitude remained larger relative to same-location TM (Fig. 5d-f). In the IHC region, the stereocilia motion amplitude was $80 \pm 11 \mathrm{~nm}$ compared to $25 \pm$ $4 \mathrm{~nm}$ for same-location TM (Fig. 5d, blue vs. red traces; mean \pm s.e.m.; $n=21$ same-location TM and IHCs from nine preparations; $p=0.0005$, Wilcoxon signed-rank test). In the row 2 OHC region, the stereocilia motion amplitude was $45 \pm 7 \mathrm{~nm}$ compared to $28 \pm 4 \mathrm{~nm}$ for same-region TM (Fig. 5e, blue vs. red traces; mean \pm s.e.m.; $n=23$ same-location TM and row 2 OHCs from 12 preparations; $p=0.009$, Wilcoxon signed-rank test). In the row $3 \mathrm{OHC}$ region, the stereocilia motion amplitude was $54 \pm$ $13 \mathrm{~nm}$ compared to $38 \pm 9 \mathrm{~nm}$ for same-region TM (Fig. 5f, blue vs. red traces; mean \pm s.e.m.; $n=17$ same-location TM and row 3 OHCs from 13 preparations; $p=0.36$, Wilcoxon signed-rank test). The larger IHC radial response is consistent with the fact that the cochlear amplifier boosts the IHC motion radially ${ }^{43}$.

Remarkably, the stereocilia radial motion BF compared poorly to that of the TM (Fig. 5d-f). In IHCs, the BF was $230 \mathrm{~Hz}$ for the stereocilia, compared to $270 \mathrm{~Hz}$ for same-location TM (Fig. 5d). In the row $2 \mathrm{OHC}$ region, the stereocilia $\mathrm{BF}$ was $200 \mathrm{~Hz}$ compared to $270 \mathrm{~Hz}$ for same-location TM (Fig. 5e). In the row 3 $\mathrm{OHC}$ region, however, both the stereocilia and same-location TM had an identical BF of $270 \mathrm{~Hz}$ (Fig. 5f), and the higher BF could be explained by the larger distance from the pivot point. However, the radial motion $\mathrm{BF}$ was clearly not a good discriminator that distinguished the IHCs from the OHCs.

A key argument frequently advanced by supporters of the IHC stimulation by viscous drag is that the IHC vs. OHC motions exhibit a phase difference as large as $180^{\circ 15,44}$, which would cause the IHCs and OHCs as well as the associated TM regions to move antiphasically $44-46$.

To address this issue, we examined the motion phases for the stereocilia bundle tip and same-location TM for frequencies between 150 and $500 \mathrm{~Hz}$ (Fig. 5g-i). The phase data were then analyzed with linear mixed modeling (LMM, see Methods).

For the IHC stereocilia bundle tip, the phase decreased from $-1.61 \pm 0.09$ to $-10.89 \pm 0.08 \mathrm{rad}$ (mean \pm s.e.m.; $n=21$ samelocation TM and IHCs from nine preparations; Fig. $5 \mathrm{~g}$, blue trace). For same-location TM, the phase decreased from $-1.56 \pm$ 0.08 to $-10.51 \pm 0.19 \mathrm{rad}$ (mean \pm s.e.m.; $n=21$ same-location TM and IHCs from nine preparations; Fig. $5 \mathrm{~g}$, red trace). The two traces were similar ( $p=0.1, \mathrm{LMM}, n=21$ same-location TM and IHCs from nine preparations). The phase-frequency function had a slope of $-0.024 \mathrm{rad} / \mathrm{Hz}$ and the frequency dependency of the phase was statistically significant $\left(p=6 \times 10^{-211}\right.$, LMM, $n=21$ same-location TM and IHCs from nine preparations).

In the row $2 \mathrm{OHC}$ region, the stereocilia bundle and TM phases decreased from $-1.71 \pm 0.08$ to $-10.91 \pm 0.11 \mathrm{rad}$ (mean \pm s.e.m.; $n=23$ same-location TM and row 2 OHCs from 12 preparations; Fig. $5 \mathrm{~h}$, blue trace) and from $-1.75 \pm 0.07$ to $-10.83 \pm 0.11 \mathrm{rad}$ (mean \pm s.e.m.; $n=23$ same-location TM and 

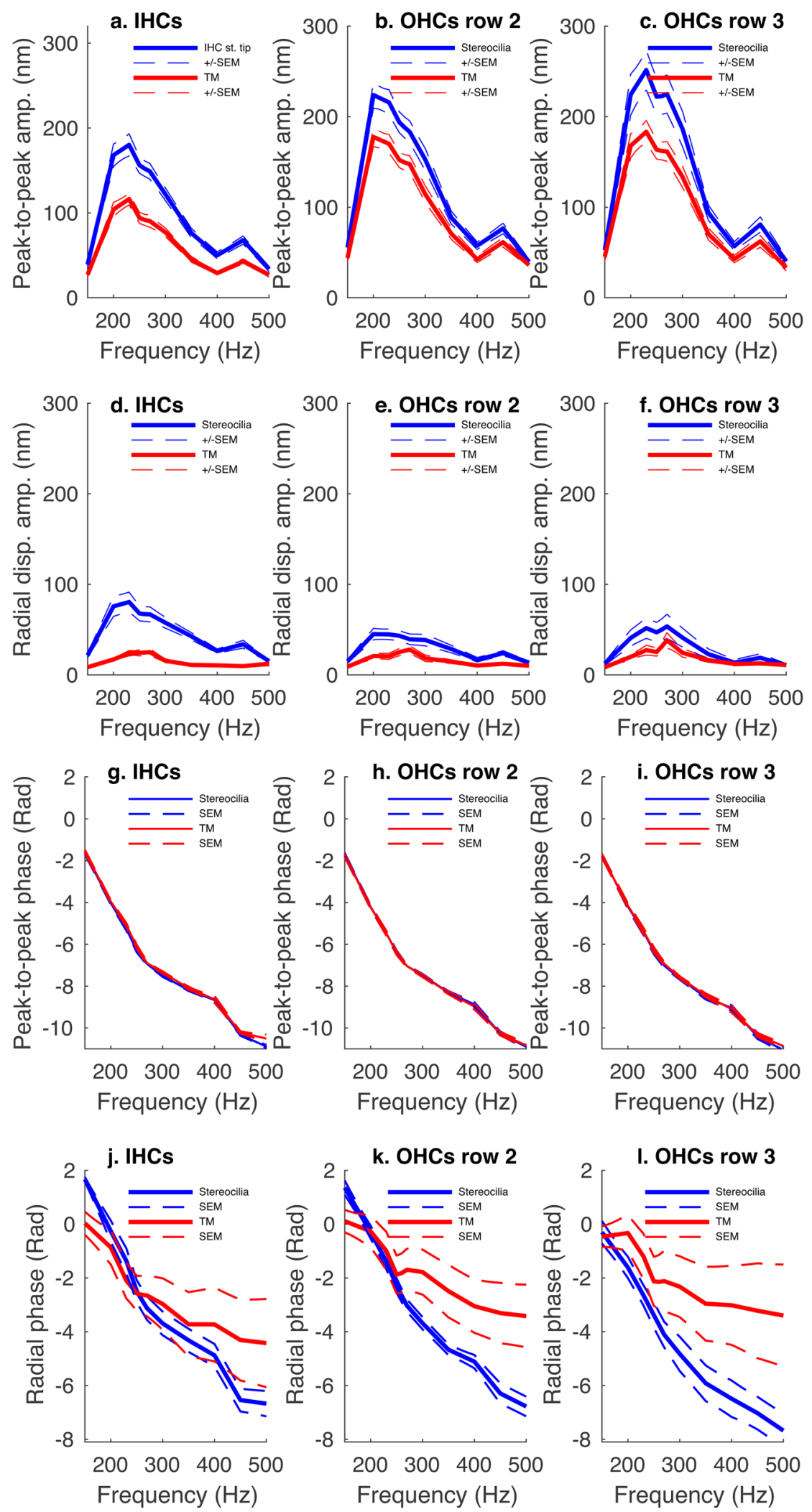

row 2 OHCs from 12 preparations; Fig. 5h, red trace), respectively. The two phase-frequency functions overlapped ( $p=0.8$, LMM, $n=23$ same-location TM and row 2 OHCs from 12 preparations). The associated slope was $-0.024 \mathrm{rad} / \mathrm{Hz}$ and the frequency dependency of the phase was statistically significant $\left(p=1 \times 10^{-232}\right.$, LMM, $n=23$ same-location TM and row 2 OHCs from 12 preparations).
In the row $3 \mathrm{OHC}$ region, the phase for the stereocilia bundle and TM decreased from $-1.76 \pm 0.09$ to $-11.06 \pm 0.15 \mathrm{rad}$ (mean \pm s.e.m. $n=17$ same-location TM and row $3 \mathrm{OHCs}$ from 13 preparations; Fig. 5i, blue trace) and from $-1.77 \pm 0.09$ to -10.87 $\pm 0.14 \mathrm{rad}$ (mean \pm s.e.m. $n=17$ same-location TM and row 3 OHCs from 13 preparations; Fig. 5i, red trace), respectively. The two phase-frequency functions were undistinguishable $(p=0.5$, 
Fig. 5 Frequency dependency of sound-evoked motion amplitude and phase of the TM and same-location IHC and OHC stereocilia bundles. a-c Peakto-peak motion amplitude for the TM (red) and same-region stereocilia (blue) computed from the associated trajectories (see Fig. 4f-h) and across frequencies (solid lines, mean; error bars, s.e.m.). Source data are provided as a Source data file. d-f Radial motion amplitude vs. frequency as computed from the trajectories described above (solid lines, mean; error bars, s.e.m.). Source data are provided as a Source data file. g-i Frequency dependency motion phases of the TM (red) and same-location stereocilia (blue) as computed along the major axis of their respective trajectories described above (solid lines, mean; error bars, s.e.m). Source data are provided as a Source data file. $\mathbf{j}-\mathbf{I}$ Same as above except that the phase plotted was the one associated with the radial component of the motion (solid lines, mean; error bar, s.e.m.). Detailed information about the $n$ values is given in the main text. Source data are provided as a Source data file.

LMM, $n=17$ same-location TM and row 3 OHCs from 13 preparations) and the associated slope was $-0.024 \mathrm{rad} / \mathrm{Hz}$. The frequency dependency of the phase was statistically significant ( $p=2 \times 10^{-177}$, LMM, $n=17$ same-location TM and row 3 OHCs from 13 preparations).

These results show that during acoustic stimulation, IHC and OHC stereocilia experience a similar mechanical relationship with TM and are consistent with the stereocilia-TM-embedment in both the IHC and OHC regions.

Mechanical measurement on isolated TM fragments from the mice have suggested that the TM has its own longitudinally propagating traveling waves ${ }^{47,48}$ that could stimulate stereocilia radially to activate the MET channels. However, this has yet to be demonstrated in a functional cochlea.

To address this issue, we evaluated the radial motion phases of the stereocilia bundle and same-location TM for frequencies between 150 and $500 \mathrm{~Hz}$ (Fig. 5j-l). The data were then analyzed by LMM.

In the IHC region, the stereocilia phase decreased from $1.67 \pm$ $0.08 \mathrm{rad}$ to $-6.67 \pm 0.47 \mathrm{rad}$ with a slope of $-0.0231 \mathrm{rad} / \mathrm{Hz}$ (mean \pm s.e.m.; $n=21$ same-location TM and IHCs from nine preparations; Fig. 5j, blue trace). For same-location TM, the phase decreased from $0.034 \pm 0.43$ to $-4.42 \pm 1.64$ rad with a slope of $0.0124 \mathrm{rad} / \mathrm{Hz}$ (mean \pm s.e.m.; $n=21$ same-location $\mathrm{TM}$ and IHCs from nine preparations; Fig. 5j, red trace). The slope difference between the two phase-frequency functions was statistically significant ( $p=0.0001, \mathrm{LMM}, n=21$ same-location TM and IHCs from nine preparations). In addition, the phase dependency in frequency and structure type were statistically significant with $p=1 \times 10^{-28}$ and 0.001 , respectively (LMM).

In the row $2 \mathrm{OHC}$ region, the phase associated to the stereocilia decreased with a slope of $-0.0228 \mathrm{rad} / \mathrm{Hz}$ from $1.36 \pm 0.27$ to $-6.78 \pm 0.37 \mathrm{rad}$ (mean \pm s.e.m.; $n=23$ same-location $\mathrm{TM}$ and row 2 OHCs from 12 preparations; Fig. 5k, blue trace). For the same-location TM, the phase decreased with a slope of -0.0108 $\mathrm{rad} / \mathrm{Hz}$ from $0.11 \pm 0.42$ to $-3.42 \pm 1.12 \mathrm{rad}$ (mean \pm s.e.m.; $n=$ 23 same-location TM and row 2 OHCs from 12 preparations; Fig. 5k, red trace). The slopes associated with the two phasefrequency functions were statistically different $\left(p=2 \times 10^{-7}\right.$, LMM, $n=23$ same-location TM and row 2 OHCs from 12 preparations). In addition, the effect of the frequency on the phase was statistically significant $\left(p=2 \times 10^{-36}\right.$, LMM).

In the row $3 \mathrm{OHC}$ region, the phase associated to the stereocilia bundle decreased with a slope of $-0.0212 \mathrm{rad} / \mathrm{Hz}$ from $-0.30 \pm$ 0.42 to $-7.68 \pm 0.63$ (mean \pm s.e.m.; $n=17$ same-location TM and row 3 OHCs from 13 preparations; Fig. 5l, blue trace). For the TM, the phase decreased with a slope $-0.0095 \mathrm{rad} / \mathrm{Hz}$ from $-0.46 \pm 0.38$ to $-3.40 \pm 1.90 \mathrm{rad}$ (mean \pm s.e.m.; $n=17$ samelocation TM and row 3 OHCs from 13 preparations; Fig. 5l, red trace). These slopes were statistically different $(p=0.0002, \mathrm{LMM}$, $n=17$ same-location TM and row 3 OHCs from 13 preparations). In addition, the effect of the frequency and structure type on the phase were statistically significant ( $p=5 \times 10^{-19}$, LMM).

Although various types of traveling waves have been shown to exist in the cochlea ${ }^{49-52}$, the radial phase data above constitute the first in situ demonstration for the existence of the TM own traveling waves ${ }^{47}$.
How deep are the IHC stereocilia-TM-embedded? To determine how deep the TM-stereocilia embedment was, we stained the OC with dextran-conjugated zFluor. According to the supplier (AAT Bioquest, USA), zFluor is much more photostable i.e., brighter than Cy5 for identical spectral characteristics.

To our surprise, zFluor produced not only a strong labeling in the TM but also in the hair cell membrane as well as their stereocilia and cuticular plates (Fig. 6a-d).

Historically, only potentiometric dyes have been known to stain hair cells ${ }^{53-55}$. That zFluor produced a strong labeling both in the hair cell membranes, their cuticular plates and stereocilia suggests that zFluor could be a reliable potentiometric dye.

A close examination of the TM region revealed that zFluor stained the Hensen's stripe as well as GSs similar to the ones seen in the confocal images of the di-3-aneppdhq-stained TM (Fig. 4). Remarkably, some of these GSs localized as low as the level of IHC cuticular plates, as seen in di-3-aneppdhq-stained TM images (compare Figs. 4a and 6), indicating that the full length of the IHC and $\mathrm{OHC}$ stereocilia bundles were embedded within the TM. Furthermore, zFluor stained TM structures that greatly resembled the $\mathrm{Ca}^{2+}$ ducts and which contacted the IHC and $\mathrm{OHC}$ stereocilia in a similar fashion as $\mathrm{Ca}^{2+}$ ducts (Fig. 6).

If zFluor stained the hair cells due to their membrane electrical properties as argued above, the staining of the GSs and $\mathrm{Ca}^{2+}$ ducts could reflect the existence of a unique electrical micro-environment in these structures, presumably conferred by $\mathrm{Ca}^{2+}$

Overall, these zFluor imaging data are consistent with the data above that showed that the TM remained attached to the IHC stereocilia even when acoustically stimulated at $150-500 \mathrm{~Hz}$.

To determine whether the TM-stereocilia links remained stable at even higher frequencies, we stimulated IHC stereocilia and same-location TM at $1 \mathrm{kHz}$ and visualized the motion by highspeed confocal imaging ${ }^{42}$. This is $5 \mathrm{x}$ the $\mathrm{BF}$ for the cochlear region of interest (Fig. 5a-f). To ensure that these low frequency hearing structures responded with a visible motion to such a high frequency, the SPL was increased to $103 \mathrm{~dB}$ SPL.

As shown by the Supplementary Movie 3 and Fig. 6d, which is a still image extracted from this movie, the TM and the IHC stereocilia moved with different motion patterns while the stereocilia bundle remained fully embedded in the TM, which is consistent with the data described above.

To investigate whether the GSs were regularly distributed along the cochlear spiral, we systematically acquired confocal images of the zFluor-stained TM and IHC stereocilia (see Fig. 6a, c, d for the exact frame size) every single $\mu \mathrm{m}$ and up to $14-35 \mu \mathrm{m}$ in five animals, giving us access to a total of 130 optical sections for the TM-IHC regions for GS quantification. Of these sections, 116 (or about $90 \%$ of them) had at least one GS and only 14 of them lacked a GS. In average, the number of GSs was $2.1 \pm 0.1$ per optical section (mean \pm s.e.m.). These data suggest that the GSs were somewhat regularly distributed longitudinally.

We note that GS-like structures have been observed by immunogold electron microscopy in the TM from the guinea pig, with irregular size and position distribution, while some of them formed clusters that could be consistent with our GSs ${ }^{56}$. 

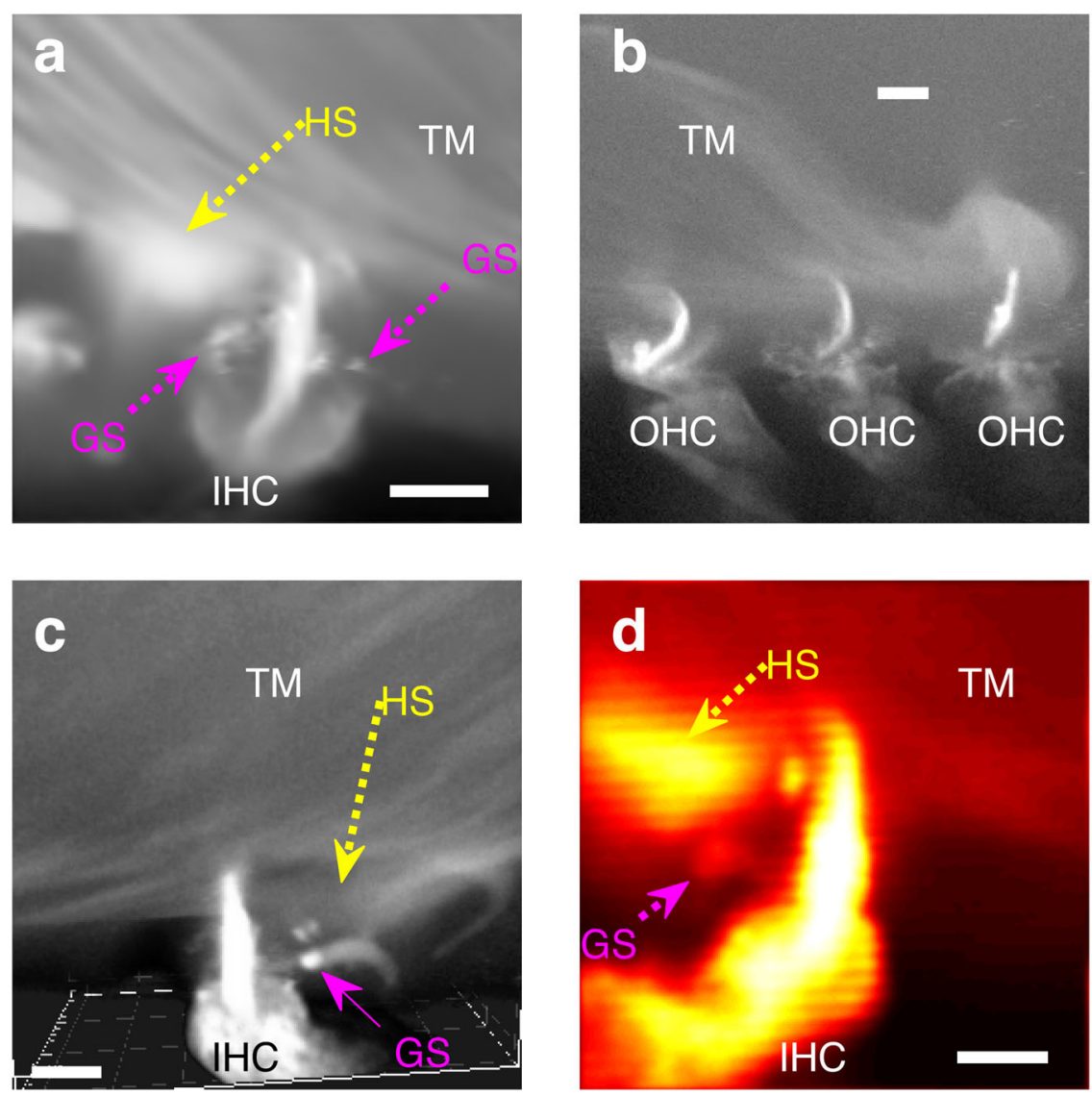

Fig. 6 IHC stereocilia are fully TM-embedded. $\mathbf{a}$, $\mathbf{b}$ The confocal images of the (dextran-conjugated) zFluor-stained OC show that zFluor stained the IHCs and $\mathrm{OHCs}$ as well as the TM. In addition, the zFluor fluorescence patterns revealed filamentous structures that contacted the stereocilia in similar fashion as the $\mathrm{Ca}^{2+}$ ducts, suggesting that the $\mathrm{Ca}^{2+}$ ducts had electrical properties that activated zFluor. The dye stained also the granular structures (GSs) as well as the Hensen's stripe (HS) most likely due to the associated electrical environments. $\mathbf{c} 3 \mathrm{D}$ reconstruction of the IHC and the associated TM region. The 3D structure was rotated to show the opposite side of the view seen in a. Again, the GSs and $\mathrm{Ca}^{2+}$ duct-like structures can easily be visualized. In addition, some GSs surprisingly localized close to the cell's cuticular plate, which is part of the RL, as seen in Fig. 4a, supporting that the TM-embedded the entire length of the IHC stereocilia bundles. The 3D reconstruction was prepared in Imaris 9.2 from 35 z-stacks with a z-step of $1 \mu$ m. $\mathbf{d}$ High-speed confocal image of the IHC stereocilia and the associated TM region captured during acoustic stimulation with a $1 \mathrm{kHz}$-tone, i.e., $5 \mathrm{x}$ their best frequency, and at 103 dB SPL. Again, the IHC stereocilia bundle can be seen TM-embedded even when stimulated with such a high frequency tone. The GS captured between the HS and the stereocilia bundle suggests that some GSs could have a connective role that links the HS to the base and tip of the stereocilia bundle. Data in a-d were representative of five different preparations. All scalebars $5 \mu \mathrm{m}$.

\section{Discussion}

In this study we have shown that $\mathrm{Ca}^{2+}$ ducts connect the TM to the stereocilia of IHCs and OHCs and that the TM-RL interface was tighter than previously thought. Specifically, the stereocilia of IHCs and OHCs were similarly TM-embedded. This similarity in stereocilia-TM relationship across hair cell types caused the IHC and OHC stereocilia to be phase-locked during sound stimulation. The IHC stereocilia remained TM-embedded even when the stimulus frequency was five times the $\mathrm{BF}$, suggesting that the IHC stereocilia-TM links are stable at high frequencies.

Phylogenetically speaking, the emergence of TM elements some 320 million years ago has generally improved the hair cell frequency sensitivity compared to TM-less OCs ${ }^{57}$.

However, the TM tends to be more useful for high frequency than for low frequency hearing ${ }^{57}$, raising the question of why amniotes conserved the TM in their low frequency hearing regions if it was barely relevant?

The presence of $\mathrm{Ca}^{2+}$ ducts and the TM-stereocilia embedment enabling the TM to convey $\mathrm{Ca}^{2+}$ and mechanical stimuli directly to the hair cells could make the TM relevant for both low and high frequency hearing regions alike.
The presence of $\mathrm{Ca}^{2+}$ ducts could also address a prominent dichotomy that has long dodged the stereocilia: the resting MET channel $\mathrm{P}_{\text {open }}$ requires 40 to $150 \mu \mathrm{M}$ in $\left[\mathrm{Ca}^{2+}\right]^{11}$, which is substantially higher than available in endolymph (typically $20 \mu \mathrm{M})^{10,58}$. $\mathrm{Ca}^{2+}$ ducts could resolve this issue by directly funnelling high TM $\mathrm{Ca}^{2+}$ concentration to the stereocilia.

Interestingly, $\mathrm{Ca}^{2+}$ removal weakened and disrupted the attachments of the TM to the RL and stereocilia. It is already known that proteins such as stereocilin attach the TM to the OHC stereocilia ${ }^{12}$. However, the expression of otoancorin near the IHC cuticular plate ${ }^{59}$ suggests that this could be one of the proteins that attach the IHC stereocilia to the TM. Our study suggests that the function of these proteins could be $\mathrm{Ca}^{2+}$ dependent and will most likely spur interest into the $\mathrm{Ca}^{2+}$ dependency of their function.

While the exact molecular nature of the $\mathrm{Ca}^{2+}$ ducts will certainly be addressed by future studies, we also expect that their discovery will inspire research into the physiological role of the $\mathrm{TM}$, whose function has been until recently thought to be mostly mechanical. 


\section{Methods}

The temporal bone preparation from the guinea pig. This study complied with all relevant ethical regulations for animal testing and research. All the animal procedures described in this study were approved by the Regional Ethics Committee in Linköping, Sweden (Permit number DNR 5111-2019).

Young adult guinea pigs of either sex (Dunkin Hartley, 200-400 g i.e., 2 to 5 weeks old) were anesthetized with a sodium pentobarbital intraperitoneal injection $(0.8 \mathrm{~mL}, 50 \mathrm{mg} / \mathrm{mL})$ and then decapitated.

Immediately, the temporal bone was carefully and rapidly excised and mounted in a custom-built chamber, after which the bulla was gently opened with a fine scalpel and scissors in order to gain access to the cochlea. Right after this step, the entire preparation was immersed in an oxygenated $\left(95 \% \mathrm{O}_{2}\right.$ and $\left.5 \% \mathrm{CO}_{2}\right)$ cell culture medium (minimum essential medium with Earle's balanced salts, room temperature). Because of the special design of the custom experimental chamber, the cochlea and the middle ear were immersed in the medium while the outer ear was shielded to allow for natural sound stimulation with a computer-controlled speaker inserted in the ear canal. With a fine scalpel, two small openings were made at the apex and base of the cochlea ${ }^{10}$. A plastic tube was then fitted into the basal opening to allow perfusion of scala tympani with the medium above gently flowing $(\sim 0.6 \mathrm{ml} / \mathrm{h})$ from an external $(4 \mathrm{ml})$ tank. The apical opening allowed the perfusion medium to flow out and was used for confocal imaging and electrophysiological recording of sound-evoked responses of OC. Because of the immersion of the middle ear, the effective sound stimulus intensity decreased by at least $20 \mathrm{~dB}$ SPL 23 . The values given in the present paper were therefore corrected accordingly.

Electrophysiology recording. For the recording of CM responses (Ix1 amplifier (Dagan Instruments) of the OC, a thin capillary glass (World Precision Instruments) was pulled with a standard puller and filled with an endolymph-mimicking solution containing $1.3 \mathrm{mM} \mathrm{NaCl}, 31 \mathrm{mM} \mathrm{KHCO}, 128.3 \mathrm{mM} \mathrm{KCl}$, and $0.023 \mathrm{mM}$ $\mathrm{CaCl}_{2}$ (pH 7.4, osmolality $300 \mathrm{mOs} / \mathrm{kg}$ ) as well as a fluorescent dye or EGTA $(100 \mu \mathrm{M})$ where indicated and bevelled at an angle of $20^{\circ}$ to $3-7 \mathrm{M} \Omega$. The electrode was then made to carefully penetrate the Reissner's membrane with a stepping manipulator. Dye and pharmacological agents dissolved in the electrolyte as above were administrated either electrophoretically or by a gentle pressure injection, where indicated, with a Picospritzer. The CM data acquisition was achieved using custom LabView software.

Confocal imaging. The OC was imaged in the preparation above with a laser scanning confocal microscope (LSM 780 (ZEN 2012 black edition software), Zeiss, Germany). The microscope and the preparation are housed in a light protected Faraday enclosure stabilized by a vibration-damping table. A $40 \mathrm{x}, 0.8 \mathrm{NA}$ water immersion lens (Nikon NIR APO) was used for reflected light confocal imaging of the $\mathrm{OC}$ and $\mathrm{Ca}^{2+}$ fluorescence confocal imaging with the ratiometric indicator Cal520L /Cy5-Dextran Conjugate also known as RatioWorks ${ }^{\text {TM }}$ (Cal-520L: excitation, $498 \mathrm{~nm}$; emission, $521 \mathrm{~nm}$; Cy5: excitation $651 \mathrm{~nm}$; emission, $670 \mathrm{~nm}$ ) as well the dextran-conjugated zFluor, where indicated (AAT Bioquest, USA). For soundevoked motion measurements by high-speed confocal imaging ${ }^{42}$, the 0.8 -NA water immersed lens was used after staining the stereocilia along with the TM with the membrane dye di-3-aneppdhq ${ }^{10}$ (Thermo Fischer).

When imaged under the cross-section view, the hair cells appear as a single IHC and three OHCs. In terms of coordinate, this z-position is not far from the injection site because this is generally where the electrode can smoothly penetrate the Reissner's membrane i.e., when it is perpendicular to the Reissner's membrane and parallel and close to the stria vascularis. Because the preparation rests on a rotative mount, this orientation is easy to find when the preparation is tilted such that the apical cochlear opening is optimally oriented relative to the lens.

For $\mathrm{Ca}^{2+}$ ratiometric imaging, Cal-520L/Cy5 dextran conjugate $(40 \mu \mathrm{M}$ in artificial endolymph) was loaded into a thin glass microelectrode. The indicator solution was then released from the electrode by application of a small pressure on the back end of the electrode ( $<4$ psi, electrode impedance $3 \mathrm{M} \Omega$ ) upon which the indicator rapidly partitioned in the TM and other water-accessible spaces from the injection site.

For $\mathrm{Ca}^{2+}$ ratio quantification for the stereocilia bundles, we used the MATLABbuilt in "drawpolygon" function to delimit the stereocilia in the reference images with successive clicks of the mouse until the contour of such a region of interest (ROI) is fully outlined. Then from the corresponding ratiometric images, we used the coordinates of these ROIs to extract and quantify $\mathrm{Ca}^{2+}$ ratio values for the stereocilia of the different hair cell types.

Frap experiments. For Cal-520L FRAP experiments, the data acquisition was performed with the lens above. After marking 1 micron-wide ROIs at the center of the stereocilia bundle and in the $\mathrm{Ca}^{2+}$ duct-rich region nearby, 50 pre-bleaching images were acquired in fast scanning mode with no interframe delay before the ROIs were photobleached with maximum laser power and then immediately 300 post-bleaching images were acquired in fast scanning mode with no interframe delay. The FRAP data were then processed in MATLAB and individual FRAP traces were fitted with a single-phase exponential model ${ }^{60}$ for statistical analysis of FRAP data in different preparations.
Sound-evoked motion analysis and trajectory fitting. The procedure for obtaining the sound-evoked trajectories ${ }^{10,42}$ for different points of interest are given throughout the text. In order to reliably quantify the sound-evoked motion amplitudes, phases and other parameters of interest for the TM and stereocilia in different preparations, sound-evoked motion trajectories from each individual measurement were fitted with the ellipse equation ${ }^{61}$ in MATLAB (The MathWorks) and were also subjected to sine wave fitting with software packages already used in other publications ${ }^{10,60}$.

Quantification and statistical analysis. As the confocal microscope setup used in this study was optimized to work with the left ears, only one temporal bone preparation could be used per animal. Consequently, the number of animals and the number of preparations are the same and are understood to be interchangeable throughout the manuscript. The number of individual measurements as well as the number of preparations (= number of animals) is given throughout the text. For the frequency-tuning of sound-evoked motion amplitudes and associated phases, the motion measurements were performed for the $150-500 \mathrm{~Hz}$. To optimize the frequency resolution especially near the BF where the motion is largest, the frequency steps were reduced to $20 \mathrm{~Hz}$ closer to the $\mathrm{BF}$ and maintained at $50 \mathrm{~Hz}$ outside this region. For figure plotting, the data were then subjected to the interp1 MATLAB function for interpolation every $10 \mathrm{~Hz}$. Data processing, analysis, and quantification were performed using custom scripts in MATLAB (The MathWorks), unless indicated otherwise.

Different types of statistic tests were used throughout this study depending on the data sets considered.

The statistical analysis of the phase across frequency for the motion of the stereocilia and same-location TM structure types was performed on the data before the interpolation step above. Accordingly, it was necessary to take into account the fact that such measurements were performed repeatedly for these structures for several frequencies. Inevitably, such repetitions introduce correlations that have to be dealt with by $\mathrm{LMM}^{62}$. The random effect in the model was the experiment ID whereas the fixed effects were the frequency and structure type. The dependent variable was the phase. The same model was used for the different hair cell regions. Calculations were performed using the lme4 and nlme packages in RStudio (version 1.2.1335) ${ }^{63}$.

For statistical analysis of motion amplitudes of the stereocilia and same-location TM at a single frequency (i.e., the BF) and FRAP parameters for the IHC stereocilia and same-location TM, the kind of correlation issue described above doesn't arise here. Consequently, for such situations, Wilcoxon signed-rank test was deemed appropriate for paired samples ${ }^{25}$, where indicated. For statistical analysis of $\mathrm{Ca}^{2+}$ ratios in the four different stereocilia types, Kruskal-Wallis test was used. These calculations were performed in MATLAB (The MathWorks).

Reporting Summary. Further information on research design is available in the Nature Research Reporting Summary linked to this article.

\section{Data availability}

All relevant data that support the findings of this study are included within this published article and/or its Supplementary Movies and the Source Data File. Any remaining data supporting the findings from this study are available from the corresponding author upon reasonable request. Source data are provided with this paper.

Received: 22 July 2020; Accepted: 6 April 2021; Published online: 10 May 2021

\section{References}

1. Denk, W., Holt, J. R., Shepherd, G. M. \& Corey, D. P. Calcium imaging of single stereocilia in hair cells: localization of transduction channels at both ends of tip links. Neuron 15, 1311-1321 (1995)

2. Kawashima, Y. et al. Mechanotransduction in mouse inner ear hair cells requires transmembrane channel-like genes. J. Clin. Invest. 121, 4796-4809 (2011).

3. Pan, B. et al. TMC1 and TMC2 are components of the mechanotransduction channel in hair cells of the mammalian inner ear. Neuron 79, 504-515 (2013).

4. Hudspeth, A. J. \& Corey, D. P. Sensitivity, polarity, and conductance change in the response of vertebrate hair cells to controlled mechanical stimuli. Proc. Natl Acad. Sci. USA 74, 2407-2411 (1977).

5. Corey, D. P. \& Hudspeth, A. J. Ionic basis of the receptor potential in a vertebrate hair cell. Nature 281, 675-677 (1979).

6. Gale, J. E. \& Ashmore, J. F. An intrinsic frequency limit to the cochlear amplifier. Nature 389, 63-66 (1997).

7. Liberman, M. C. et al. Prestin is required for electromotility of the outer hair cell and for the cochlear amplifier. Nature 419, 300-304 (2002).

8. Issa, N. P. \& Hudspeth, A. J. The entry and clearance of $\mathrm{Ca} 2+$ at individual presynaptic active zones of hair cells from the bullfrog's sacculus. Proc. Natl Acad. Sci. USA 93, 9527-9532 (1996). 
9. Yu, X. L., Lewis, E. R. \& Feld, D. Seismic and auditory tuning curves from bullfrog saccular and amphibian papillar axons. J. Comp. Physiol. A 169, 241-248 (1991).

10. Strimbu, C. E., Prasad, S., Hakizimana, P. \& Fridberger, A. Control of hearing sensitivity by tectorial membrane calcium. Proc. Natl Acad. Sci. U.S.A 116, 5756-5764 (2019).

11. Goldring, A. C., Beurg, M. \& Fettiplace, R. The contribution of TMC1 to adaptation of mechanoelectrical transduction channels in cochlear outer hair cells. J. Physiol. 597, 5949-5961 (2019).

12. Verpy, E. et al. Stereocilin-deficient mice reveal the origin of cochlear waveform distortions. Nature 456, 255-258 (2008)

13. Von Békésy, G. \& Wever, E. G. Experiments in Hearing (Acoustical Society of America, 1989).

14. Rhode, W. S. \& Geisler, C. D. Model of the displacement between opposing points on the tectorial membrane and reticular lamina. J. Acoust. Soc. Am. 42, 185-190 (1967).

15. Dallos, P., Billone, N. C., Durrant, J. D., Wang, C.-Y. \& Raynor, S. Cochlear inner and outer hair cells: functional differences. Science 177, 356-358 (1972).

16. Russell, I. J. \& Sellick, P. M. Low-frequency characteristics of intracellularly recorded receptor potentials in guinea-pig cochlear hair cells. J. Physiol. 338, 179-206 (1983).

17. Cheatham, M. A. \& Dallos, P. Response phase: a view from the inner hair cell. J. Acoust. Soc. Am. 105, 799-810 (1999).

18. Dallos, P., Santos-Sacchi, J. \& Flock, A. Intracellular recordings from cochlear outer hair cells. Science 218, 582-584 (1982).

19. Bredberg, G., Lindeman, H. H., Ades, H. W., West, R. \& Engström, H. Scanning electron microscopy of the organ of corti. Science 170, 861-863 (1970).

20. Raufer, S., Guinan, J. J. \& Nakajima, H. H. Cochlear partition anatomy and motion in humans differ from the classic view of mammals. Proc. Natl Acad. Sci. USA 116, 13977-13982 (2019).

21. Lukashkin, A. N. et al. A mouse model for human deafness DFNB22 reveals that hearing impairment is due to a loss of inner hair cell stimulation. Proc. Natl Acad. Sci. USA 109, 19351-19356 (2012).

22. Ulfendahl, M., Flock, A. \& Khanna, S. M. Isolated cochlea preparation for the study of cellular vibrations and motility. Acta Otolaryngol. Suppl. 467, 91-96 (1989).

23. Ulfendahl, M. et al. Mechanical response characteristics of the hearing organ in the low-frequency regions of the cochlea. J. Neurophysiol. 76, 3850-3862 (1996).

24. Fridberger, A., Tomo, I., Ulfendahl, M. \& Boutet de Monvel, J. Imaging hair cell transduction at the speed of sound: dynamic behavior of mammalian stereocilia. Proc. Natl Acad. Sci. USA 103, 1918-1923 (2006).

25. Hakizimana, P., Brownell, W. E., Jacob, S. \& Fridberger, A. Sound-induced length changes in outer hair cell stereocilia. Nat. Commun. 3, 1094 (2012).

26. Fridberger, A. \& de Monvel, J. B. Sound-induced differential motion within the hearing organ. Nat. Neurosci. 6, 446-448 (2003).

27. Honrubia, V. \& Ward, P. H. Longitudinal distribution of the cochlear microphonics inside the cochlear duct (guinea pig). J. Acoust. Soc. Am. 44, 951-958 (1968).

28. Warren, R. L. et al. Minimal basilar membrane motion in low-frequency hearing. Proc. Natl Acad. Sci. USA 113, E4304-E4310 (2016)

29. Tomo, I. et al. Imaging the living inner ear using intravital confocal microscopy. NeuroImage 35, 1393-1400 (2007).

30. Richter, C.-P., Emadi, G., Getnick, G., Quesnel, A. \& Dallos, P. Tectorial membrane stiffness gradients. Biophys. J. 93, 2265-2276 (2007)

31. Verpy, E. et al. Stereocilin connects outer hair cell stereocilia to one another and to the tectorial membrane. J. Comp. Neurol. 519, 194-210 (2011).

32. Flock, A., Flock, B., Fridberger, A., Scarfone, E. \& Ulfendahl, M. Supporting cells contribute to control of hearing sensitivity. J. Neurosci. J. Soc. Neurosci. 19, 4498-4507 (1999).

33. Hackney, C. M. \& Furness, D. N. The composition and role of cross links in mechanoelectrical transduction in vertebrate sensory hair cells. J. Cell Sci. 126, 1721-1731 (2013).

34. Siemens, J. et al. Cadherin 23 is a component of the tip link in hair-cell stereocilia. Nature 428, 950-955 (2004).

35. Kronester-Frei, A. The effect of changes in endolymphatic ion concentrations on the tectorial membrane. Hear. Res. 1, 81-94 (1979).

36. Shah, D. M., Freeman, D. M. \& Weiss, T. F. The osmotic response of the isolated, unfixed mouse tectorial membrane to isosmotic solutions: effect of $\mathrm{Na}+, \mathrm{K}+$, and $\mathrm{Ca} 2+$ concentration. Hear. Res. 87, 187-207 (1995).

37. Michel, V. et al. Cadherin 23 is a component of the transient lateral links in the developing hair bundles of cochlear sensory cells. Dev. Biol. 280, 281-294 (2005).

38. Flock, A., Bretscher, A. \& Weber, K. Immunohistochemical localization of several cytoskeletal proteins in inner ear sensory and supporting cells. Hear. Res. 7, 75-89 (1982).

39. Ballesteros, A., Fenollar-Ferrer, C. \& Swartz, K. J. Structural relationship between the putative hair cell mechanotransduction channel TMC1 and TMEM16 proteins. eLife 7, e38433 (2018).
40. Lippincott-Schwartz, J. \& Patterson, G. H. Development and use of fluorescent protein markers in living cells. Science 300, 87-91 (2003).

41. Ghaffari, R., Page, S. L., Farrahi, S., Sellon, J. B. \& Freeman, D. M. Electrokinetic properties of the mammalian tectorial membrane. Proc. Natl Acad. Sci. USA 110, 4279-4284 (2013).

42. Jacob, S., Tomo, I., Fridberger, A., de Monvel, J. B. \& Ulfendahl, M. Rapid confocal imaging for measuring sound-induced motion of the hearing organ in the apical region. J. Biomed. Opt. 12, 021005 (2007).

43. Tomo, I., Boutet de Monvel, J. \& Fridberger, A. Sound-evoked radial strain in the hearing organ. Biophys. J. 93, 3279-3284 (2007).

44. Nowotny, M. \& Gummer, A. W. Nanomechanics of the subtectorial space caused by electromechanics of cochlear outer hair cells. Proc. Natl Acad. Sci. USA 103, 2120-2125 (2006).

45. Ramamoorthy, S. et al. Filtering of acoustic signals within the hearing organ. J. Neurosci. 34, 9051-9058 (2014).

46. Jacob, S., Pienkowski, M. \& Fridberger, A. The endocochlear potential alters cochlear micromechanics. Biophys. J. 100, 2586-2594 (2011).

47. Ghaffari, R., Aranyosi, A. J. \& Freeman, D. M. Longitudinally propagating traveling waves of the mammalian tectorial membrane. Proc. Natl Acad. Sci. USA 104, 16510-16515 (2007).

48. Ghaffari, R., Aranyosi, A. J., Richardson, G. P. \& Freeman, D. M. Tectorial membrane travelling waves underlie abnormal hearing in Tectb mutant mice. Nat. Commun. 1, 96 (2010).

49. Cooper, N. P. \& Rhode, W. S. Nonlinear mechanics at the apex of the guineapig cochlea. Hear. Res. 82, 225-243 (1995).

50. Cooper, N. P. \& Rhode, W. S. Fast travelling waves, slow travelling waves and their interactions in experimental studies of apical cochlear mechanics. Auditory Neuroscience 2, 289-299 (1996).

51. Temchin, A. N., Recio-Spinoso, A., Cai, H. \& Ruggero, M. A. Traveling waves on the organ of corti of the chinchilla cochlea: spatial trajectories of inner hair cell depolarization inferred from responses of auditory-nerve fibers. $J$. Neurosci. 32, 10522-10529 (2012).

52. Robles, L., Rhode, W. S. \& Geisler, C. D. Transient response of the basilar membrane measured in squirrel monkeys using the Mössbauer effect. $J$. Acoust. Soc. Am. 59, 926-939 (1976).

53. Nakagawa, T., Oghalai, J. S., Saggau, P., Rabbitt, R. D. \& Brownell, W. E. Photometric recording of transmembrane potential in outer hair cells. $J$. Neural. Eng. 3, 79-86 (2006).

54. Ramamoorthy, S., Wilson, T. M., Wu, T. \& Nuttall, A. L. Non-uniform distribution of outer hair cell transmembrane potential induced by extracellular electric field. Biophys. J. 105, 2666-2675 (2013).

55. Fisher, J. A. N., Kowalik, L. \& Hudspeth, A. J. Imaging electrical resonance in hair cells. Proc. Natl Acad. Sci. USA 108, 1651-1656 (2011).

56. Andrade, L. R., Salles, F. T., Grati, M., Manor, U. \& Kachar, B. Tectorins crosslink type II collagen fibrils and connect the tectorial membrane to the spiral limbus. J. Struct. Biol. 194, 139-146 (2016).

57. Manley, G. A. Cochlear mechanisms from a phylogenetic viewpoint. Proc. Natl Acad. Sci. USA 97, 11736-11743 (2000).

58. Bosher, S. K. \& Warren, R. L. Very low calcium content of cochlear endolymph, an extracellular fluid. Nature 273, 377-378 (1978).

59. Zwaenepoel, I. et al. Otoancorin, an inner ear protein restricted to the interface between the apical surface of sensory epithelia and their overlying acellular gels, is defective in autosomal recessive deafness DFNB22. Proc. Nat Acad. Sci. USA 99, 6240-6245 (2002).

60. Prasad, S. et al. Radixin modulates the function of outer hair cell stereocilia. Commun. Biol. 3, 1-15 (2020).

61. Ohad Gal. Fit_ellipse. https://www.mathworks.com/matlabcentral/ fileexchange/3215-fit_ellipse (2003).

62. Bagger-Sjöbäck, D. et al. High-frequency hearing, tinnitus, and patient satisfaction with stapedotomy: a randomized prospective study. Sci. Rep. 5, 13341 (2015).

63. RStudio Team. RStudio: Integrated Development for R. (RStudio, Inc., 2018).

\section{Acknowledgements}

We thank J. Plue for discussions regarding linear mixed modeling of the data and S. Hedin for drawing the cartoon representation of the OC presented as Fig. $3 \mathrm{f}$ and for reading the manuscript. The work was funded by grants from Tysta Skolan Foundation (to P.H.), the Swedish research council grants (2018-02692 and 2017-06092), and the US National Institutes of Health (to A.F.).

\section{Author contributions}

P.H. designed research, performed research, and analysed the data. P.H. and A.F. interpreted the data. P.H. and A.F. prepared the manuscript.

\section{Funding}

Open access funding provided by Linköping University. 


\section{Competing interests}

The authors declare no competing interests.

\section{Additional information}

Supplementary information The online version contains supplementary material available at https://doi.org/10.1038/s41467-021-22870-1.

Correspondence and requests for materials should be addressed to P.H.

Peer review information Nature Communications thanks the anonymous reviewer(s) for their contribution to the peer review of this work.

Reprints and permission information is available at http://www.nature.com/reprints

Publisher's note Springer Nature remains neutral with regard to jurisdictional claims in published maps and institutional affiliations. (c) (i) Open Access This article is licensed under a Creative Commons Attribution 4.0 International License, which permits use, sharing, adaptation, distribution and reproduction in any medium or format, as long as you give appropriate credit to the original author(s) and the source, provide a link to the Creative Commons license, and indicate if changes were made. The images or other third party material in this article are included in the article's Creative Commons license, unless indicated otherwise in a credit line to the material. If material is not included in the article's Creative Commons license and your intended use is not permitted by statutory regulation or exceeds the permitted use, you will need to obtain permission directly from the copyright holder. To view a copy of this license, visit http://creativecommons.org/ licenses/by/4.0/.

(C) The Author(s) 2021, corrected publication 2021 